\title{
Eight-Months After Anterior Cruciate Ligament Reconstruction: Is It Time to Return to Physical Activity?
}

\author{
Saulė Salatkaitė, Laimonas Šiupšinskas, \\ Renata Žumbakytė-Šermukšnienè, Rimtautas Gudas \\ Lithuanian University of Health Sciences, Kaunas, Lithuania
}

\begin{abstract}
Background. Return to participation in sport is an important outcome when evaluating the success of anterior cruciate ligament (ACL) reconstruction surgery. A review by Arden et al. (2014) demonstrates that just because an athlete has surgery, it does not automatically mean that they will return to sport. So, the purpose of this study was to assess if patients could return to their pre-injury activity level 8 months after ACLR.

Methods. Patients completed IKDC-2000, ACL-RSI questionnaires, underwent FMS, YBT, HOP test sets and drop jump tests, which were evaluated with the LESS. ACL laxity was measured by using a GNRB® device. Participants were 81 patients. They were divided into two groups based on single leg HOP test results. The first group included 30 persons who all had their four single leg HOP test scores above $90 \%$. The second group consisted of 51 persons for whom at least one of the single leg HOP tests scored lower than $90 \%$.

Results. IKDC and HOP test results in the first group scored higher than in the second group. Psychological confidence was higher in the first group, who had better HOP test results. For the first group, FMS and LESS results were better than those for the second group. Moderate positive correlation was found between LESS scores and laxity results, FMS, three HOP sub-tests. IKDC had the biggest number of correlations with other tests. Results showed negative and weak correlation with laxity results using $134 \mathrm{~N}$ power, as well as positive and moderate correlation with HOP sub-tests, FMS, ACL-RSI. Single HOP for distance, triple HOP for distance, crossover HOP for distance and LESS increased the odds of achieving RTS criteria. No other factors were found to be associated with the accomplishment of reaching RTS criteria after ACLR.

Conclusions. The main factor which can help decide if a person can return to their pre-injury level of physical activity are the single leg HOP test and LESS. The use of single leg HOP tests and LESS along with psychological and self-reported evaluations is the best test combination for assessment 8 months after ACLR.
\end{abstract}

Keywords: ACL reconstruction, function tests, return to play, jump tests, psychological readiness.

\section{INTRODUCTION}

$\mathrm{T}$ The aim for most athletes and physically active people who undergo an anterior cruciate ligament reconstruction (ACLR) is to rehabilitate their full knee ability, thus allowing them to return to sport practice (Ebert et al., 2018). However, up to $16-33 \%$ of athletes who return to their previous level of sport activity with ACLR suffer a second anterior cruciate ligament (ACL) injury (Wiggins et al., 2016). The following ACL injuries can be a re-tearing of the formerly injured $\mathrm{ACL}$ or a later injury to the contralateral ACL
(Schilaty et al., 2017). Moreover, only 55\% of athletes return to previous levels of sports activity after ACLR (Ardern et al., 2014). Therefore, determining factors that increase the probability of returning to sports activity (RTS) would be useful. Plenty of factors and limitations can have an affect on returning to sports activity after ACLR and current knowledge regarding these factors is restricted (Cristiani et al., 2020). Previous reports suggest that one of the best predictive factors in returning to sports activity is single leg HOP tests 
with an optimal cut-off value of $81.3 \%$ (Kitaguchi et al., 2019). Also, other researchers found that single leg HOP tests were strongly associated with quadriceps strength. The capability of quadriceps to generate torque quickly is one of the major factors in achieving dynamic knee stability during highimpact movements such as jumping or hopping (Pua, Mentiplay Clark, \& Ho 2017). Thus, it can be assumed that single leg HOP tests help to qualitatively assess the strength of the operated leg. However, RTS can be determined not only by the functioning of the knee joint, muscle strength, but also by the psychological condition of patients.

Ardern et al. (2013) reported that psychological responses are important as well as one leg HOP tests' symmetry and subjective knee function to RTS. Up to $78 \%$ of athletes who did not have RTS claimed they feared re-injury (Kitaguchi et al., 2019). Also, researchers could not find any differences between sexes. Athletes (male and female) demonstrated parallel trends in readiness to RTS before and after surgery (Kostyun, Burland, Kostyun, Milewski, \& Nissen, 2019). So we can see how necessary it is to assess readiness to return to sports activity and confidence during recovery. They may help discover those persons who are at risk of re-injury.

The purpose of this study was to assess if patients could return to pre-injury activity levels 8 months after ACL reconstruction. We hypothesized that a combination of physical measures and selfreported outcomes would enable us to discover deficits in patients after ACLR.

\section{METHODS}

The study was conducted in the Department of Sports Medicine at the Lithuanian University of Health Sciences between 2016 and 2020. This study was approved by the Regional Biomedical Research Ethics Committee, No. BE-2-24. Every participant signed a written consent form.

Participants. This study included patients who underwent ACL reconstruction. The inclusion criteria were: 18 years-old, capacity to do all functional tests, no pain or swelling in the knee during tests, no other injury or surgery in the other or same leg. Exclusion criteria were: younger than 18 years-old, past surgery or injury in the other leg, other musculoskeletal injuries or illnesses which may have influence on tests results.

Research methods. All patients completed International Knee Documentation Committee
(IKDC) and ACL Return to Sport after Injury (ACL-RSI) questionnaires, underwent functional movement screen tests (FMS), Y balance tests (YBT), HOP test sets and drop jump tests, which were evaluated using the Landing Error Scoring System (LESS). Also, for all participants, ACL laxity was measured by using the Genourob device.

The ACL-RSI questionnaire was developed to measure psychological factors associated with returning to sports activity following ACLR surgery. It evaluates emotions, performance and risk. Additionally, this questionnaire was translated and adapted into Lithuanian (Salatkaite, Šiupšinskas, \& Gudas, 2019). The FMS comprises seven fundamental movement patterns (deep squat, hurdle step, in-line lunge, shoulder mobility, active straight leg rise, trunk stability push-up, and rotary stability), which require balance between mobility and stability. Each test is undertaken for one specific movement which requires appropriate functioning of the body's kinetic linking system (Cook, Burton, \& Hoogenboom, 2006a,b). The YBT is used to measure physical performance, compare balance ability and identify individuals who have chronic ankle or knee instability. The device utilizes the anterior, posteromedial and posterolateral components. The maximal reach distance is measured by reading the tape measure at the edge of the reach indicator, at the point where the most distal part of the foot reached (Plisky et al., 2009). The LESS score is simply a count of landing technique "errors" on a range of readily observable items of human movement. A higher LESS score indicates poor technique in landing from a jump; a lower LESS score indicates better jump-landing technique (Padua et al., 2009). The HOP test set consists of a single HOP for distance, a $6 \mathrm{~m}$ HOP for time, a triple HOP for distance and a crossover HOP for distance. All tests were performed twice, with the average of the two values used to calculate limb symmetry (Noyes, Barber, \& Mangine, 1991). The GNRB ${ }^{\circledR}$ is a knee laxity testing device for measuring anteroposterior tibial translation at $20^{\circ}$ of knee flexion, thus reproducing the Lachman test position. A linear jack gradually increases thrust forces according to the examiner, at: $67,89,134$, 150 or $250 \mathrm{~N}$ on the upper section of the calf. A displacement transducer $(0.1 \mathrm{~mm}$ precision) records the relative displacement of the anterior tibial tubercle with respect to the femur. Motion data obtained from the displacement transducer produce a force-displacement curve whose slope determines 
ligamentous elasticity (Robert, Nouveau, Gageot, \& Gagnière, 2009).

Statistical analysis. Descriptive statistics were calculated for all outcomes and are presented as the mean (standard deviation) value for continuous variables. Comparisons between groups were performed using the Mann-Whitney $U$ test and results are presented as the median (interquartile range) value. Correlations between LESS, FMS, IKDC and other subjective/objective evaluation methods were analyzed using Pearson or Spearman correlation coefficients. The Pearson correlation was used when each pair of variables was bivariately normally distributed. In other cases, the Spearman correlation was used. The linear correlations were interpreted as follows: .5-1.0 as strong; .3-.5 as moderate; and $.1-.3$ as weak. Multivariate binary logistic regression analyses were performed with single leg HOP test sets, LESS, YBT, FMS test performances, IKDC and ACL-RSI questionnaire answers as independent variables, as well as LSI >
$90 \%$ as passing RTS scores as dependent variable (coded $0 ; 1$ ). Independent variables were rejected until the model became correct. All statistical analyses were performed using the SPSS version 20 (IBM Corp, Armonk, New York, USA). $p<.05$ was considered statistically significant.

\section{RESULTS}

The study participants were 81 patients (51 males and 30 females) who underwent ACLR. The participants were divided into two groups based on single leg HOP test results. The first group comprised 30 persons ( 10 females, 20 males), whose four single leg HOP test results scored above 90\% of limb symmetry index (LSI). The second group held 51 persons ( 20 females, 31 males) for whom least one single leg HOP test result scored lower than $90 \%$ of LSI. The patients were tested starting 6 months after ACLR. The descriptive data for all subjects is presented in Table 1.

\begin{tabular}{|c|c|c|c|c|}
\hline \multirow{5}{*}{$\begin{array}{l}\text { Table 1. Patient demo- } \\
\text { graphics }\end{array}$} & & LSI $>90 \%{ }^{a}$ & LSI $\leq 90 \%{ }^{a}$ & $p$-value \\
\hline & Age (years old) & $22.3(5.34)$ & $24.7(7.54)$ & .10 \\
\hline & Height $(\mathrm{cm})$ & $183.67(12.79)$ & $181.04(10.66)$ & .35 \\
\hline & Weight $(\mathrm{kg})$ & $78.18(15.17)$ & $80.32(15.04)$ & .54 \\
\hline & BMI & $22.98(2.35)$ & $24.36(3.17)$ & .18 \\
\hline \multirow{2}{*}{$\begin{array}{l}\text { Note. }{ }^{\text {a }} \text { Data is shown as } \\
\text { the mean (standard de- } \\
\text { viation) value }\end{array}$} & Graft size & $10.02(0.99)$ & $10.17(1.01)$ & .50 \\
\hline & Time from ACLR to testing (months) & $8.87(3.00)$ & $8.35(2.53)$ & .77 \\
\hline
\end{tabular}

Statistically significant differences were found between the groups' tests and questionnaire results (Table 2). The IKDC questionnaire result for the first group was 10.4 points higher than that of the second group. Also, all single leg HOP test results were higher in the first group (single HOP for distance LSI - 9.6\% difference; triple HOP for distance LSI $-6.97 \%$ difference; crossover HOP for distance LSI - 11.35\% difference, $6 \mathrm{~m}$ HOP for time LSI - 6.81\% difference). Psychological confidence, as shown in the results, was higher in the first group, who had better single leg HOP test results (score was 15\% higher). Also, the first group's FMS and LESS were better than those of the second group (18 vs $15 ; 5$ vs 5 , respectively).

Correlations were found between LESS, FMS, IKDC and other tests, and they are presented in Tables 3, 4 and 5. Moderate and positive correlation was found between the LESS score and laxity results. Moderate and positive correlation was found between FMS and three single leg HOP sub-tests. The IKDC questionnaire had the largest number of correlations with other tests. Results showed negative and weak correlation with laxity results using $134 \mathrm{~N}$ power, positive and moderate correlation with single leg HOP sub-tests, FMS and the ACL-RSI questionnaire.

The multivariate binary logistic regression model was used to test the contribution of the HOP test set, LESS, YBT, FMS, IKDC and ACL-RSI in predicting the possibility of returning to sports activity. After the first run of the multivariate binary logistic regression model, we found that YBT, FMS, IKDC and ACL-RSI were not important predictor variables. The analysis was re-run without these unimportant variables. In the second analysis, we found that the single HOP for distance, triple HOP for distance, crossover HOP for distance and LESS scores increased the odds of achieving an LSI $>90 \%$ (Table 6). 
Table 2. Knee function tests and questionnaire scores for each group

\begin{tabular}{|c|c|c|c|}
\hline & LSI $>90 \% \%^{a}$ & $\mathrm{LSI} \leq 90 \% \mathrm{a}^{\mathrm{a}}$ & $p$-value \\
\hline Laxity Slope P2 & $4.7(2.3 ; 8.3)$ & $3.6(1.8 ; 5.75)$ & .74 \\
\hline Laxity $134 \mathrm{~N}$ displ. Difference (mm) & $0.4(-04 ; 1.4)$ & $0.5(0 ; 1.25)$ & .35 \\
\hline Laxity 134 N Dmax difference (mm) & $0.4(-0.4 ; 1.4)$ & $0.5(0 ; 1.25)$ & .35 \\
\hline Laxity $150 \mathrm{~N}$ displ. Difference (mm) & $0.5(-0.5 ; 1.2)$ & $0.6(-0.05 ; 1.25)$ & .07 \\
\hline Laxity 150 N Dmax difference (mm) & $0.6(-0.4 ; 1.2)$ & $0.6(0 ; 1.35)$ & .09 \\
\hline IKDC score (pt) & $89.7(80.45 ; 96.85)$ & $79.3(73 ; 85.65)$ & $.01 *$ \\
\hline Single HOP for distance LSI (\%) & $96.55(92.45 ; 102.59)$ & $86.95(79.87 ; 90.33)$ & $<.001 *$ \\
\hline Crossover HOP for distance LSI (\%) & $100.48(94.74 ; 102.41)$ & $89.13(80.81 ; 92.27)$ & $<.001 *$ \\
\hline Triple HOP for distance LSI (\%) & $94.47(95.96 ; 102.09)$ & $87.5(82.77 ; 90.51)$ & $<.001 *$ \\
\hline 6m HOP for time LSI (\%) & $99.47(95.42 ; 101.02)$ & $92.66(85.86 ; 96.44)$ & $<.001 *$ \\
\hline YBT anterior difference $(\mathrm{cm})$ & $-2(-4.38 ; 1.13)$ & $-2.5(-7.5 ; 0)$ & .09 \\
\hline YBT posteromedial difference $(\mathrm{cm})$ & $-0.5(-2.5 ; 3.13)$ & $-2.5(-7.5 ; 1)$ & $.01 *$ \\
\hline YBT posterolateral difference $(\mathrm{cm})$ & $-0.5(-3.63 ; 2.75)$ & $0(-5.5 ; 2)$ & .71 \\
\hline YBT composite score left & $101.06(91.29 ; 106.89)$ & 97.31 (92.91; 102.12) & .29 \\
\hline YBT composite score right & $99.54(91.46 ; 105.98)$ & $96.17(92.88 ; 102.04)$ & .24 \\
\hline FMS score $(p t)$ & $18(14.75 ; 18)$ & $15(12 ; 17)$ & $.004 *$ \\
\hline ACL-RSI score $(\%)$ & $85(68.13 ; 90.83)$ & $70(46.67 ; 85)$ & $.046^{*}$ \\
\hline LESS score (pt) & $5(4 ; 6)$ & $7(5 ; 8)$ & $<.001 *$ \\
\hline
\end{tabular}

Notes. "Significant difference between groups, $p<.05$. 'Data is shown as the median (interquartile range) value.

Table 3. Correlations between LESS, FMS, IKDC and laxity results

Notes. $r$ - Correlation coefficient; "Significant difference, $p<.05$

\begin{tabular}{|l|c|c|c|c|}
\hline & $\begin{array}{c}\text { Laxity 134N displ. } \\
\text { difference }\end{array}$ & $\begin{array}{c}\text { Laxity 134N Dmax } \\
\text { difference }\end{array}$ & $\begin{array}{c}\text { Laxity 150N displ. } \\
\text { difference }\end{array}$ & $\begin{array}{c}\text { Laxity 150N Dmax } \\
\text { difference }\end{array}$ \\
\hline LESS score & $r=.301$ & $r=.301$ & $r=.317$ & $r=.344$ \\
$.02 *$ & $.02 *$ & $.01 *$ & $.01 *$ \\
\hline \multirow{2}{*}{ FMS score } & $r=-.118$ & $r=-.118$ & $r=-.024$ & .61 \\
\hline \multirow{2}{*}{ IKDC score } & .32 & .32 & $r=-.06$ \\
& $r=-.265$ & $r=-.265$ & .17 & $r=-.151$ \\
\hline
\end{tabular}

Table 4. Correlations between LESS, FMS, IKDC and single leg HOP test results

Notes. $r$ - Correlation coefficient; *Significant difference, $p<.05$

\begin{tabular}{|l|c|c|c|}
\hline & $\begin{array}{c}\text { Single HOP for distance } \\
\text { LSI }\end{array}$ & $\begin{array}{c}\text { Crossover HOP for } \\
\text { distance LSI }\end{array}$ & $\begin{array}{c}\text { Triple HOP for distance } \\
\text { LSI }\end{array}$ \\
\hline LESS score & $r=-.284$ & $r=-.310$ & $r=-.183$ \\
$.02 *$ & $.01 *$ & .09 \\
\hline FMS score & $r=.432$ & $r=.262$ & $r=.284$ \\
& $<.001 *$ & $.02 *$ & $.01 *$ \\
\hline IKDC score & $r=.451$ & $r=.505$ & $r=.439$ \\
& $<.001 *$ & $<.001 *$ & $.001 *$ \\
\hline
\end{tabular}


Table 5. Correlations between LESS, FMS, IKDC and YBT test and ACL-RSI questionnaire results

\begin{tabular}{|l|l|l|l|l|l|c|}
\hline & $\begin{array}{c}\text { YBT anterior } \\
\text { difference }\end{array}$ & $\begin{array}{c}\text { YBT posteromedial } \\
\text { difference }\end{array}$ & $\begin{array}{c}\text { YBT composite } \\
\text { score left }\end{array}$ & $\begin{array}{c}\text { YBT composite } \\
\text { score right }\end{array}$ & \multicolumn{1}{|c|}{$\begin{array}{c}\text { FMS } \\
\text { score }\end{array}$} & $\begin{array}{c}\text { ACL-RSI\% } \\
\text { score }\end{array}$ \\
\hline \multirow{2}{*}{ LESS score } & $r=-.119$ & $r=-.198$ & $r=-.320$ & $r=-.323$ & $r=-.453$ & $r=-.298$ \\
& .28 & .06 & $.01 *$ & .07 & $<.001 *$ & $.01 *$ \\
\hline \multirow{2}{*}{ FMS score } & $r=.075$ & $r=.017$ & $r=.289$ & $r=.298$ & & $r=.199$ \\
& .47 & .87 & $.01 *$ & $.01 *$ & - & .05 \\
\hline \multirow{2}{*}{ IKDC score } & $r=.315$ & $r=.303$ & $r=.023$ & $r=.108$ & $r=.314$ & $r=.541$ \\
& $.02 *$ & $.02 *$ & .84 & .35 & $.02 *$ & $<.001 *$ \\
\hline
\end{tabular}

Notes. $r$ - Correlation coefficient; "Significant difference, $p<.05$

Table 6. Factors affecting the achievement of a pass result of RTS (LSI > 90\%) after ACL reconstruction

Notes. $\mathrm{R}^{2}=0.855$ (Nagelkerke). Model $\mathrm{x}^{2}(5)=48.47, p<.001$. Correct model classification $93.2 \%$. SE standard error; OR odds ratio; CI confidence interval *Significant difference between groups, $p<.05$

\begin{tabular}{|l|c|c|l|c|}
\hline & $\begin{array}{c}\text { Regression } \\
\text { coefficient (B) }\end{array}$ & SE & \multicolumn{1}{|c|}{ OR (95\% CI) } & $p$-value \\
\hline Single HOP for distance LSI & 0.293 & 0.125 & $\begin{array}{l}1.341 \\
(1.05-1.712)\end{array}$ & $.02 *$ \\
\hline Triple HOP for distance LSI & 0.330 & 0.112 & $\begin{array}{l}1.391 \\
(1.116-1.734)\end{array}$ & $.003 *$ \\
\hline Crossover HOP for distance LSI & 0.248 & 0.109 & $\begin{array}{l}1.282 \\
(1.035-1.587)\end{array}$ & $.02 *$ \\
\hline LESS & 0.216 & 0.105 & $\begin{array}{l}1.241 \\
(1.011-1.524)\end{array}$ & $.04 *$ \\
\hline
\end{tabular}

\section{DISCUSSION}

The main finding of this study was that the best RTS factor predictors were single HOP for distance, triple HOP for distance and LESS tests. According to other authors, one of the strongest RTS predictive factors is LSI in the single leg HOP test (Kitaguchi et al., 2019). These discoveries determined why we decided to compare our test results between groups, divided by single leg HOP test results. The most interesting findings were that if a patient had lower than $90 \%$ as a single leg HOP test result, we could say that the results of the FMS or LESS tests would be worse, and that the person would have less confidence in his/her knee function. According to Ithurburn, Longfellow, Thomas, Paterno, and Schmitt (2019), patients who went back to pre-injury activity levels had better results for the involved limb on the single leg HOP tests than those who did not return to sports activity. Also, other authors found that taking into account the single leg HOP tests and ACLRSI questionnaire results together, just $37.9 \%$ of patients were ready to return to pre-injury activity levels (Meierbachtol, Yungtum, Paur, Bottoms, \&
Chmielewski, 2018). The present study allowed us to see that higher single leg HOP test results can be associated with superior psychological readiness.

These authors have studied the mechanisms of ACL injury in depth. The most common mechanism of non-contact ACL injury involves a significant dynamic valgus rotational force directed onto a fixed planted foot (Alentorn-Geli et al., 2009). Clinical recommendations have begun to include LESS as a component of comprehensive criteria that may be used to identify common mechanisms of ACL injury (Welling et al., 2018). Our preliminary study showed that the LESS score could help predict ACL laxity. Researchers demonstrated that movement quality was also a predictive biomechanical risk factor of non-contact or indirect-contact ACL injury (Padua et al., 2015). We can conclude that higher ACL laxity affects jump biomechanics, which can be a cause of not returning to pre-injury physical activity levels.

Besides the relationship between physical impairments and RTS, evidence is emerging that psychological responses are strong predictors 
associated with RTS rates following athletic injury (Arden et al., 2013). In this research, significant differences were found between groups in ACLRSI and IKDC tests. Some authors state that psychological readiness and patient self-reported outcomes are not associated with physical function and knee laxity (Webster, Nagelli, Hewett, \& Feller, 2018). However, this study revealed moderate correlation amongst the IKDC, ACL-RSI and single leg HOP, and LESS tests. So, it can be concluded that poor physical function determines low psychological response.

Recent studies have demonstrated an increased re-injury risk if patients do not meet objective test LSIs within testing batteries of $>90 \%$ before RTS, inclusion of functional HOP and strength assessments (Ebert et al., 2018). In the present study there were 30 people who reached the RTS pass criteria after almost 9 months from surgery. Nonetheless, Gokeler and his co-authors (2017) found that at 6 months after ACLR there were no patients who scored LSI $>90 \%$. The current data indicates that performance on the single HOP for distance test, triple HOP for distance test and LESS may be good indicators of the risk of future ACL injuries after ACLR and RTS. The single leg RTS tests and LESS can be performed in a relatively short timeframe with minimal cost and technical skills. Nevertheless, it is presently not known what the clinical appropriate cut off point is in order for the LESS to determine patients who are not fully recovered.
There are some limitations in our study. It included young patients who were operated on by the same surgeon at the same hospital. This may explain why the results are not very generalized. Secondly, there was a small number of patients included in this study. In order to demonstrate the chances of ACL re-rupture, a larger group of subjects is needed. Additionally, after carrying out the series of RTS tests, there is now a lack of information about patients' re-injuries after examination. This remains a focus point for our future work.

\section{CONCLUSIONS}

The evidence that emerges from this study suggests that patients 8 months after ACLR and willing to go back to pre-injury activity levels require additional rehabilitation and training. The main factors which can help to decide if a person can return to pre-injury physical activity levels are the single leg HOP test and LESS. Also, the use of single leg HOP tests and LESS, along with psychological and self-reported evaluation, is the best test combination for passing rate assessment 8 months after ACLR.

Conflict of interest statement. No conflict of interest is declared.

Funding. No funding was received for this study.

\section{REFERENCES}

Alentorn-Geli, E., Myer, G. D., Silvers, H. J., Samitier, G., Romero D., Lázaro-Haro, C., \& Cugat R. (2009). Prevention of noncontact anterior cruciate ligament injuries in soccer players. Part 1: Mechanisms of injury and underlying risk factors. Knee Surgery, Sports Traumatology, Arthroscopy, 17(7), 705-729. doi: 10.1007/s00167-009-0813-1.

Arden, C. L., Taylor, N. F., Feller, J. A., Whitehead, T. S., \& Webster, K. E. (2013). Psychological responses matter in returning to preinjury level of sport after anterior cruciate ligament reconstruction surgery. The American Journal of Sports Medicine, 41(7), 1549-1558. doi: $10.1177 / 0363546513489284$.

Arden, C. L., Taylor, N. F., Feller, J. A., \& Webster, K. E. (2014). Fifty-five percent return to competitive sport following anterior cruciate ligament reconstruction surgery: an updated systematic review and meta-analysis including aspects of physical functioning and contextual factors. British Journal of Sports Medicine, 48(21), 1543-1552. doi: 10.1136/bjsports-2013-093398.
Cook, G., Burton, L., \& Hoogenboom, B. (2006a). Pre-participation screening: The use of fundamental movements as an assessment of function - Part 1. North American Journal of Sports Physical Therapy, 1(2), $62-72$.

Cook, G., Burton, L., \& Hoogenboom, B. (2006b). Pre-participation screening: the use of fundamental movements as an assessment of function - Part 2. North American Journal of Sports Physical Therapy, 1(3), $132-139$.

Cristiani, R., Mikkelsen, C., Edman, G., Forssblad, M., Engström, B., \& Stålman, A. (2020). Age, gender, quadriceps strength and hop test performance are the most important factors affecting the achievement of a patient-acceptable symptom state after ACL reconstruction. Knee Surgery, Sports Traumatology, Arthroscopy, 28, 369-380. doi: 10.1007/s00167-01905576-2.

Ebert, J. R., Edwards, P., Currie, J., Smith, A., Joss, B., Ackland, T., Buelow ,J. U., \& Hewitt, B. (2018). 
Comparison of the 'Back in Action' Test Battery to Standard Hop Tests and Isokinetic Knee Dynamometry in patients following anterior cruciate ligament reconstruction. International Journal of Sports Physical Therapy, 13(3), 389-400.

Ebert, J. R., Edwards, P., Yi, L., Joss B., Ackland, T., Carey-Smith, R., Buelow, J. U., \& Hewitt, B. (2018). Strength and functional symmetry is associated with post-operative rehabilitation in patients following anterior cruciate ligament reconstruction. Knee Surgery, Sports Traumatology, Arthroscopy, 26(8), 2353-236. doi: 10.1007/s00167-017-4712-6.

Gokeler, A., Welling, W., Zaffagnini, S., Seil, R., \& Padua, D. (2017). Development of a test battery to enhance safe return to sports after anterior cruciate ligament reconstruction. Knee Surgery, Sports Traumatology, Arthroscopy, 25(1), 192-199. doi: 10.1007/s00167-016-4246-3.

Ithurburn, M. P., Longfellow, M. A., Thomas, S., Paterno, M. V., \& Schmitt, L. C. (2019). Knee function, strength, and resumption of preinjury sports participation in young athletes following anterior cruciate ligament reconstruction. Journal of Orthopaedic \& Sports Physical Therapy, 49(3), 145-153. doi: 10.2519/jospt.2019.8624.

Kitaguchi, T., Tanaka, Y., Takeshita, S., Tsujimoto, N., Kita, K., Amano, H., Kinugasa, K., Tachibana,Y., Natsuume, T., \& Horibe, S. (2019). Importance of functional performance and psychological readiness for return to preinjury level of sports 1 year after ACL reconstruction in competitive athletes. Knee Surgery, Sports Traumatology, Arthroscopy, 28, 2203-2212. doi: 10.1007/s00167-019-05774-y.

Kostyun, R. O., Burland, J. P., Kostyun, K. J., Milewski, M. D., \& Nissen, C. W. (2019). Male and female adolescent athletes' readiness to return to sport after anterior cruciate ligament injury and reconstruction. Clinical Journal of Sports Medicine, 00, 1-5. doi: 10.1097/JSM.0000000000000751.

Meierbachtol, A., Yungtum, W., Paur, E., Bottoms, J., \& Chmielewski, T. L. (2018). Psychological and functional readiness for sport following advanced group training in patients with anterior cruciate ligament reconstruction. Journal of Orthopaedic \& Sports Physical Therapy, 48(11), 864-872. doi: 10.2519/jospt.2018.8041.

Noyes, F. R., Barber, S. D., \& Mangine, R. E. (1991). Abnormal lower limb symmetry determined by function hop tests after anterior cruciate ligament rupture. The American Journal of Sports Medicine, 19(5), 513-518. doi: 10.1177/036354659101900518.

Padua, D. A., DiStefano, L. J., Beutler, A. I., Motte, S. J., DiStefano, M. J., Steven, W., \& Marshall, S. W. (2015). The landing error scoring system as a screening tool for an anterior cruciate ligament injury-prevention program in elite-youth soccer athletes. Journal of Athletic Training, 50(6), 589-595. doi: 10.4085/1062-6050-50.1.10.
Padua, D. A., Marshall, S. W., Boling, M. C., Thigpen, C. A., Garrett, W. E., \& Beutler, A. I. (2009). The Landing Error Scoring System (LESS) is a valid and reliable clinical assessment tool of jump-landing biomechanics. The American Journal of Sports Medicine, 37(10), 1996-2002. doi: 10.1177/0363546509343200.

Plisky, P. J., Gorman, P. P., Butler, R. J., Kiesel, K. B., Underwood, F. B., \& Elkins, B. (2009). The reliability of an instrumented device for measuring components of the Star Excursion Balance Test. North American Journal of Sports Physical Therapy, 4(2), 92-99.

Pua, Y. H., Mentiplay, B. F., Clark, R. A., \& Ho, J. Y. (2017). Associations among quadriceps strength and rate-of-torque development six weeks post anterior cruciate ligament reconstruction and future hop and vertical jump performance: A prospective cohort study. Journal of Orthopaedic \& Sports Physical Therapy, 47(11), 845-852. doi: 10.2519/jospt.2017.7133.

Robert, H., Nouveau, S., Gageot, S., \& Gagnière, B. (2009). A new knee arthrometer, the GNRB $\AA$ : Experience in ACL complete and partial tears. Orthopaedics \& Traumatology: Surgery \& Research, 95(3), 171-176. doi: 10.1016/j.otsr.2009.03.009.

Salatkaitè, S., Šiupšinskas, L., \& Gudas, R. (2019). Translation and cultural adaptation of Lithuanian version of the anterior cruciate ligament return to sport after injury (ACL-RSI) scale. PLoS ONE, 14(7), 1-8. doi: 10.1371/journal.pone.0219593.

Schilaty, N. D., Nagelli, C., Bates, N. A., Sanders, T. L., Krych, A. J., Stuart, M. J., \& Hewett, T. E. (2017). Incidence of second anterior cruciate ligament tears and identification of associated risk factors from 2001-2010 using a geographic database. Orthopaedic Journal of Sports Medicine, 5(8), 1-8. doi: 10.1177/2325967117724196.

Webster, K. E., Nagelli, C. V., Hewett, T. E., \& Feller, J. A. (2018). Factors associated with psychological readiness to return to sport after anterior cruciate ligament reconstruction surgery. The American Journal of Sports Medicine, 46(7), 1545-1550. doi: 10.1177/0363546518773757.

Welling, W., Benjaminse, A., Seil, R., Lemmink, K., Zaffagnini, S., \& Gokeler, A. (2018). Low rates of patients meeting return to sport criteria 9 months after anterior cruciate ligament reconstruction: a prospective longitudinal study. Knee Surgery, Sports Traumatology, Arthroscopy, 26(12), 3636-3644. doi: 10.1007/s00167018-4916-4.

Wiggins, A. J., Grandhi, R. K., Schneider, D. K., Stanfield, D., Webster, K. E., \& Myer, G. D. (2016). Risk of secondary injury in younger athletes after anterior cruciate ligament reconstruction: A systematic review and meta-analysis. The American Journal of Sports Medicine, 44(7), 1861-1876. doi: 10.1177/0363546515621554. 